\title{
The future of immune checkpoint blockade immunotherapy: towards personalized therapy or towards combination therapy
}

\author{
Zhiwei Hu \\ Department of Surgery Division of Surgical Oncology, The Ohio State University College of Medicine, James Comprehensive Cancer Center, \\ Columbus, OH, USA \\ Contributions: Zhiwei Hu. Department of Surgery Division of Surgical Oncology, The Ohio State University College of Medicine, James \\ Comprehensive Cancer Center, 410 West 12th Avenue, Columbus, OH 43210, USA. Email: zhiwei.hu@osumc.edu. \\ Provenance: This is an invited Editorial commissioned by Section Editor Dr. Chunlin Ou (Cancer Research Institute of Central South University, \\ Changsha, China). \\ Correspondence to: Prat A, Navarro A, Paré L, et al. Immune-related gene expression profiling after PD-1 blockade in non-small cell lung carcinoma, \\ head and neck squamous cell carcinoma and melanoma. Cancer Res 2017;77:3540-50.
}

Submitted Sep 22, 2017. Accepted for publication Sep 29, 2017.

doi: $10.21037 /$ jtd.2017.10.31

View this article at: http://dx.doi.org/10.21037/jtd.2017.10.31

One of the major breakthroughs in cancer immunotherapy in the past decades was the discovery of immune checkpoint molecules, notably cytotoxic T-lymphocyte associated protein 4 (CTLA-4) (1) and the programmed cell death-1 (PD-1, also known as CD279) T-cell receptor (2) and its ligands (programmed death-ligand 1 (PD-L1, also known as B7-H1 or CD274) (3) and PD-L2 (also known as B7DC or CD273) (4). PD-1 is expressed on activated CD4 and CD8 T cells, natural killer (NK) cells, B cells, activated monocytes and dendritic cells (DC) (5). Recent clinical trials with anti-PD-1 and PD-L1 monoclonal antibodies as immune checkpoint blockades have shown unprecedented durable responses in some patients with a variety of cancers. However, only a minority of the total treated patients benefit from the current checkpoint blockade therapy. In other words, resistance to immune checkpoint antibody blockades is commonly observed in most cancer patients (6). There is a great need to better predict the clinical outcome and to overcome the resistance. To optimize the checkpoint blockage therapy, Sznol and Chen have identified several key challenges (7), including the development of more accurate predictive biomarkers of response and rational combinations of therapy.

To better predict the clinical outcome for cancer patients, Prat and colleagues recently reported in the July 1 , 2017 issue of Cancer Research (8) that PD-1 gene expression along with 12 gene signatures tracking CD4 and CD8 T cell activation, and NK cells and interferon (IFN) activation was significantly associated with better clinical outcome (referring as non-progression disease and progressionfree survival) after anti-PD-1 antibody therapy with pembrolizumab or nivolumab (both are monoclonal IgG4 antibodies against PD-1) in 65 patients with melanoma, lung non-squamous, squamous cell lung or head and neck cancers.

This interesting finding has several important implications for immune checkpoint blockade therapy. Firstly, the results suggest that patients with pre-existing infiltration of CD4 and CD8 T cells and NK cells will better benefit anti-PD-1 therapy. As the authors discussed, the method developed in their study could be potentially used as a genomic tool, in addition to the protein biomarker assays for PD-L1 (and PD-1) expression in patients' tumors by immunohistochemistry (IHC), for evaluating immunerelated gene expression profiles to select patients who are more likely to benefit from anti-PD-L1 and PD-1 blockade therapy. The authors also discussed similar findings in two additional recent studies evaluating the association of immune-related gene expression in patients with advanced melanoma (Ayers et al., Abstract presented at $30^{\text {th }}$ Annual Meeting of SITC 2015) and NSCLC ( $\mathrm{n}=224)$ (9) with antiPD-L1 or PD-1. A research article in 2017 f Clin Invest by Ayers and colleagues (10) reported that the T cell-inflamed gene expression profile contained IFN-gamma-responsive 
genes related to antigen presentation, chemokine expression, cytotoxic activity and adaptive immune resistance. However, these gene expression features were necessary, but not always sufficient, for [predicting] clinical benefit (10). Secondly, the immune gene signatures identified in these studies as being associated with anti-PD-1 or PD-L1 therapy response or survival are part of the genes previously identified as the immunological constant of rejection (ICR)related genes, such as CXCR3, CCR5 ligand genes and IFN-gamma signaling genes. As the authors emphasized, these ICR-related genes are also associated with increased survival across cancer types and responsiveness to other cancer immunotherapy approaches, including anti-CTLA4, adoptive therapy/IL2 and vaccination (8). Thirdly, the findings also suggest that any combination therapy that can increase infiltration of CD4 and CD8 T cells and/or NK cells in tumor microenvironment may enhance the efficacy of immune checkpoint blockage therapy.

Similar observations were made previously and independently by Tumeh and colleagues (11). Tumeh et al. analyzed tumor samples from 46 patients with metastatic melanoma obtained before and during anti-PD-1 therapy (pembrolizumab) using quantitative IHC, quantitative multiplex immunofluorescence, and next- generation sequencing for T-cell antigen receptors. They reported that pre-treatment samples obtained from responding patients showed higher numbers of CD8-, PD-1- and PD-L1expressing cells at the invasive tumor margin and inside tumors, suggesting that the presence of tumor-infiltrating lymphocytes is an important biomarker for predicting responses to PD-L1 blockade therapy (11).

To increase infiltration of immune cells in tumor microenvironment, one potential strategy is to use vaccines, cytokines and other existing or new immune therapy that could augment localized or systemic immunity. For example, one recent approach reported by Tang et al. (6) was to target cancer cells with tumor necrosis factor (TNF) superfamily member LIGHT, a protein ligand that can bind to two different receptors, herpes virus entry mediator (HVEM, also known as tumor necrosis factor receptor superfamily member 14, TNFRSF14), and lymphotoxin beta receptor (LTßR). Targeting LIGHT on cancer cells could overcome tumor resistance to PD-L1 blockade through activating LT $\beta R$ signaling and recruiting $T$ cell infiltration in tumor microenvironment (6).

Another potential strategy to increase infiltration of $T$ and NK cells in tumor microenvironment is to directly infuse chimeric antigen receptor-T and NK cells or ex vivo expanded NK cells. Chimeric antigen receptors (CARs) are membrane-bound synthetic receptors on $\mathrm{T}$ and $\mathrm{NK}$ (CAR-T and NK) cells to target cancer cell-surface antigens and augment $\mathrm{T}$ and NK function. CAR-T and NK cell therapy has been evaluated in hematological malignancies in clinical trials and in approved clinical applications (12) and is also being translated into solid cancers (13). This strategy will not only provide systemic immunity that is required for effective cancer immunotherapy (14), but may also increase local infiltration of $\mathrm{T}$ and NK cells in tumor microenvironment. In line with this idea, a recent review summarized the most recent clinical results on the use of checkpoint inhibitors and CAR-T cells in multiple myeloma (15). As such, multiple myeloma could be an appropriate candidate to test the idea of the combination therapy of checkpoint blockades with CAR-T therapy to increase infiltration of $\mathrm{T}$ cells in tumor microenvironment to overcome the resistance and thus to optimize the efficacy of immune checkpoint blockade therapy. To increase local infiltration of CAR-T and NK cells, we hypothesize that CAR-T or NK cells can be delivered locally or regionally, such as intracranial delivery for brain cancer and intrahepatica infusion for hepatocellular carcinoma and liver metastases from primary colorectal cancer (16). Cell therapy, including ex vivo expanded and activated NK cells, is being evaluated in clinical trials for hematological cancers as well as for solid cancers (17). To increase homing of the systemically infused NK cells to tumor microenvironment, Kremer and colleagues very recently reported (18) that genetic engineering of human NK cells to express a chemokine receptor CXCR2 improved migration to renal cell carcinoma that expresses the ligand for CXCR2.

As discussed above, there is a great need to develop additional methods and tools, in addition to IHC staining for expression of PD-1 and PD-L1, to predict biomarkers of response to checkpoint blockade therapy, such as genomic tools reported by Prat et al. (8) and Tumeh et al. (11) because clinical trials have already shown that only a minority of patients benefited from the current checkpoint blockades. What can we offer for the majority of patients if their tumors are negative for expression of these checkpoint molecules, or are too few or lack of infiltration of CD4, CD8 T cells and NK cells, or even are positive for the expression of checkpoint molecules but are still resistant to current blockade therapy? In fact, immune impairment is commonly observed in cancer patients, such as impairment of T cells (19) and NK cells (20). To achieve an optimal clinical outcome for cancer patients, we believe that 
the future of immune checkpoint blockade therapy, or in general, cancer immunotherapy, should be towards personalized combination therapy with existing approaches, such as: therapeutic antibodies, antibody-drug conjugates, immune checkpoint blockades, CAR-T and NK cells, DCs, vaccines, oncolytic viruses, cytokines and/or depletion of immune suppressor cells like myeloid-derived suppressor cells (MDSC), regulatory T cells (Treg), tumor-associated macrophages (TAM), etc. or with not-yet-discovered newer and better immunotherapies. The combination immunotherapy can target different molecules on some or all major tumor compartments, including but not limited to the cancer cells, tumor neovasculature, cancer stem cells, MDSC, Treg and TAM, or ideally, target the same molecule that is commonly expressed by these major tumor compartments. In this regard, we believe that tissue factor (also known as coagulation factor III or CD142) is one of few such promising and common target molecules as it is commonly yet selectively expressed on many types of solid cancer cells as well as leukemic cells $(21,22)$, tumor angiogenic vascular endothelial cells in vitro (23) and in vivo (22,24-26) and cancer stem cells (27).

\section{Acknowledgements}

We thank Carrie Boerio and Aaron Conley, Directors of Foundation Relations at The Ohio State University Wexner Medical Cancer, for critical reading and editing.

Funding: This work was supported by the Dr. Ralph and Marian Falk Medical Research Trust Awards Programs. The project described was also partly supported by Award Number UL1TR001070 from the National Center for Advancing Translational Sciences through a Phase 1 L-Pilot Award and a voucher award from the Ohio State University Center for Clinical and Translational Science.

\section{Footnote}

Conflicts of Interest: $\mathrm{Z} \mathrm{Hu}$ is co-inventor of the first generation tissue factor-targeting "neovascular-targeted immunoconjugates" (ICON) and is the inventor of a second and a third generation tissue factor-targeting ICONs, named L-ICON1 and L-ICON3 (Patents Pending).

\section{References}

1. Brunet JF, Denizot F, Luciani MF, et al. A new member of the immunoglobulin superfamily--CTLA-4. Nature
1987;328:267-70.

2. Ishida $Y$, Aqata $Y$, Shibahara $\mathrm{K}$, et al. Induced expression of PD-1, a novel member of the immunoglobulin gene superfamily, upon programmed cell death. EMBO J 1992;11:3887-95.

3. Dong H, Zhu G, Tamada K, et al. B7-H1, a third member of the B7 family, co-stimulates T-cell proliferation and interleukin-10 secretion. Nat Med 1999;5:1365-9.

4. Latchman Y, Wood CR, Chernova T, et al. PD-L2 is a second ligand for PD-1 and inhibits T cell activation. Nat Immunol 2001;2:261-8.

5. Keir ME, Butte MJ, Freeman GJ, et al. PD-1 and its ligands in tolerance and immunity. Annu Rev Immunol 2008;26:677-704.

6. Tang H, Wang Y, Chlewicki LK, et al. Facilitating T Cell Infiltration in Tumor Microenvironment Overcomes Resistance to PD-L1 Blockade. Cancer Cell 2016;29:285-96.

7. Sznol M, Chen L. Antagonist antibodies to PD-1 and B7H1 (PD-L1) in the treatment of advanced human cancer-response. Clin Cancer Res 2013;19:5542.

8. Prat A, Navarro A, Paré L, et al. Immune-related gene expression profiling after PD-1 Blockade in non-small cell lung carcinoma, head and neck squamous cell carcinoma, and melanoma. Cancer Res 2017;77:3540-50.

9. Fehrenbacher L, Spira A, Ballinger et al. Atezolizumab versus docetaxel for patients with previously treated non-small-cell lung cancer (POPLAR): a multicentre, open-label, phase 2 randomised controlled trial. Lancet 2016;387:1837-46.

10. Ayers M, Lunceford J, Nebozhyn M, et al. IFN- $\gamma$-related mRNA profile predicts clinical response to PD-1 blockade. J Clin Invest 2017;127:2930-40.

11. Tumeh PC, Harview CL, Yearley JH, et al. PD-1 blockade induces responses by inhibiting adaptive immune resistance. Nature 2014;515:568-71.

12. Luskin MR, DeAngelo DJ. Chimeric antigen receptor therapy in acute lymphoblastic leukemia clinical practice. Curr Hematol Malig Rep 2017. [Epub ahead of print].

13. Morello A, Sadelain M, Adusumilli PS. Mesothelintargeted CARs: driving t cells to solid tumors. Cancer Discov 2016;6:133-46.

14. Spitzer MH, Carmi Y, Reticker-Flynn NE, et al. Systemic immunity is required for effective cancer immunotherapy. Cell 2017;168:487-502.e15.

15. Gay F, D'Agostino M, Giaccone L, et al. Immuno-oncologic approaches: CAR-T Cells and checkpoint inhibitors. Clin Lymphoma Myeloma Leuk 2017;17:471-8.

16. Sridhar P, Petrocca F. Regional delivery of chimeric 
antigen receptor (CAR) T-Cells for cancer therapy.

Cancers (Basel) 2017;9:E92.

17. Baggio L, Laureano ÁM, Silla LMDR. Natural killer cell adoptive immunotherapy: Coming of age. Clin Immunol 2017;177:3-11.

18. Kremer V, Ligtenberg M, Zendehdel R, et al. Genetic engineering of human NK cells to express CXCR2 improves migration to renal cell carcinoma. J Immunother Cancer 2017;5:73.

19. Aerts JG, Hegmans JP. Tumor-specific cytotoxic T cells are crucial for efficacy of immunomodulatory antibodies in patients with lung cancer. Cancer Res 2013;73:2381-8.

20. Hu Z. Overcome the impairment of NK cells for icon and antibody immunotherapy of cancer. J Immune Based Ther Vaccines Antimicrob 2013;2:1-8.

21. $\mathrm{Hu} \mathrm{Z}$. Factor VII-targeted photodynamic therapy for breast cancer and its therapeutic potential for other solid cancers and leukemia, breast cancer - current and alternative therapeutic modalities, Esra Gunduz and Mehmet Gunduz (Ed.), ISBN: 978-953-307-776-5, InTech 2011. Available online: http://www.intechopen. com/articles/show/title/factor-vii-targeted-photodynamictherapy-for-breast-cancer-and-its-therapeutic-potentialfor-other-s

Cite this article as: $\mathrm{Hu} \mathrm{Z}$. The future of immune checkpoint blockade immunotherapy: towards personalized therapy or towards combination therapy. J Thorac Dis 2017;9(11):42264229. doi: $10.21037 /$ jtd.2017.10.31
22. Hu Z, Garen A. Targeting tissue factor on tumor vascular endothelial cells and tumor cells for immunotherapy in mouse models of prostatic cancer. Proc Natl Acad Sci U S A 2001;98:12180-5.

23. $\mathrm{Hu} \mathrm{Z}$, Cheng J, Xu J, et al. Tissue factor is an angiogenicspecific receptor for factor VII-targeted immunotherapy and photodynamic therapy. Angiogenesis 2017;20:85-96.

24. Contrino J, Hair G, Kreutzer DL, et al. In situ detection of tissue factor in vascular endothelial cells: correlation with the malignant phenotype of human breast disease. Nat Med 1996;2:209-15.

25. Cheng J, Xu J, Duanmu J, et al. Effective treatment of human lung cancer by targeting tissue factor with a factor VII-targeted photodynamic therapy. Curr Cancer Drug Targets 2011;11:1069-81.

26. Duanmu J, Cheng J, Xu J, et al. Effective treatment of chemoresistant breast cancer in vitro and in vivo by a factor VII-targeted photodynamic therapy. Br J Cancer 2011;104:1401-9.

27. $\mathrm{Hu} \mathrm{Z}, \mathrm{Xu} \mathrm{J}$, Cheng J, et al. Targeting tissue factor as a novel therapeutic oncotarget for eradication of cancer stem cells isolated from tumor cell lines, tumor xenografts and patients of breast, lung and ovarian cancer. Oncotarget 2017;8:1481-94. 